•综述・

\title{
洞察景观环境影响蜜蜂之新视角: 肠道微生物
}

\author{
唐 敏 ${ }^{1,2}$ 邹 怡 ${ }^{3}$ 苏秦之 ${ }^{2,4}$ 周 欣 ${ }^{1,2 *}$ \\ 1 (中国农业大学植物保护学院, 北京 100193) \\ 2 (中国农业大学北京食品营养与人类健康高精尖创新中心, 北京 100193) \\ 3 (西交利物浦大学健康与环境科学系, 江苏苏州 215123) \\ 4 (中国农业大学食品科学与营养工程学院, 北京 100083)
}

摘要：作为生态服务提供者的传粉蜜蜂与景观生态息息相关，而以农田为主的景观组成显著降低了传粉蜜蜂的 多样性。目前调查研究显示, 农田的扩张与蜜蜂多样性下降相关, 且农药残留对蜜蜂损害严重。景观中的开花植 物决定了蜜蜂的食物(营养)组成, 其中花粉蛋白含量与蜜蜂的生长发育紧密相关。尽管研究已证实景观环境会显 著影响蜜蜂蜂群的发展和个体的生长繁殖能力, 但未来还需要加强景观组成变化直接作用于蜜蜂的机制研究。另 一方面, 大量研究表明蜜蜂肠道共生菌是影响宿主健康的重要因素: 可促进宿主吸收营养和抵抗病原菌。作为传 粉者, 蜜蜂接触到的主要外部环境——花粉和花蜜都含有特殊的微生物, 很多研究暗示花源微生物是蜜蜂肠道菌 来源之一。研究表明景观环境相关的食物(营养)、农药残留以及环境微生物都会显著影响肠道微生物。现有少量 的研究证明不同景观的蜜蜂肠道微生物有差异, 景观环境可能通过作用于蜜蜂肠道微生物进而影响蜜蜂健康。然 而不同景观环境中的微生物, 尤其是花源微生物和蜜蜂肠道菌之间的关联有待证明。景观对蜜蜂肠道微生物的影 响值得研究, 希望可以从肠道菌的视角鉴别对蜜蜂友好的景观环境, 进而指导土地合理利用和蜜蜂保护。

关键词：景观; 蜜蜂; 肠道微生物; 农药; 花粉营养; 环境微生物

\section{A new perspective on landscape impact in bee populations: Considering the bee gut microbiome}

\author{
Min Tang ${ }^{1,2}$, Yi Zou ${ }^{3}$, Qinzhi Su ${ }^{2,4}$, Xin Zhou ${ }^{1,2^{*}}$ \\ 1 College of Plant Protection, China Agricultural University, Beijing 100193 \\ 2 Beijing Advanced Innovation Center for Food Nutrition and Human Health, China Agricultural University, Beijing 100193 \\ 3 Department of Health Environmental Sciences, Xi'an Jiaotong-Liverpool University, Suzhou, Jiangsu 215123 \\ 4 College of Food Science and Nutrtional Engineering, China Agricultural University, Beijing 100083
}

\begin{abstract}
Pollinator bees are providers of an important ecosystem service, and their survival relies completely on the landscape. Now with the landscape dominated by agriculture, bee diversity has been significantly reduced. Studies suggest that bee populations decline as agricultural land-use increases due to increased exposure to detrimental pesticides. Further, the protein content of pollen is highly important for the growth and development of a bee, and different landscapes provide distinct sources of nutrition. Although many studies have demonstrated the apparent impacts of landscape change on the population dynamics and individual survival of the bees, the underpinning mechanisms remain largely unknown. On the other hand, an increasing body of literature has shown that bee gut symbionts are of great importance to the health of the host bees in absorbing nutrients and resisting pathogens. When foraging, pollinator bees are exposed to particular microbes from pollen and nectar which have been suggested to be a source of some bee gut symbionts and could be either probiotics or pathogens. Together with landscape-related nutrition and pesticides, environmental microbes have been reported to affect bee microbiomes significantly. A number of pilot studies suggest that landscape change could affect bee microbiota, thereby influencing host health. An important linkage, however, is missing between environmental microbiota, especially those associated with
\end{abstract}

收稿日期: 2019-03-12; 接受日期: 2019-05-27

基金项目: 国家自然科学基金(31772493)和科技基础资源调查专项(2018FY100403)

* 通讯作者 Author for correspondence. E-mail: xinzhoucaddis@icloud.com 
the flowers, and that of the bee gut in a changing habitat. It is worth exploring how gut microbiomes respond to landscape changes. This will hopefully help us identify landscape types that are friendly to bees, so proper land-use can be implemented to protect the bees.

Key words: landscape; bees; gut microbiome; pesticide; pollen nutrition; environmental microbes

传粉昆虫在生态系统和农业生产中扮演着重 要的角色(Klein et al, 2007; Gill et al, 2016), 80\%以 上的开花植物需要动物, 绝大多数是昆虫帮助授粉 (Kremen et al, 2007; Ollerton et al, 2011)。近年来, 由 于人类活动的影响, 自然景观生态发生了巨大的变 化, 有研究发现土地利用变化是导致生物多样性降 低的主要原因之一(Murphy \& Romanuk, 2014)。其 中农业用地的大面积扩展破坏了原有的自然、半自 然生境, 大规模的集约化农业使得生境多样性降低 (Ollerton et al, 2014; Ramankutty et al, 2018), 威胁 着传粉昆虫及其生态服务功能 (Biesmeijer et al, 2006; Burkle et al, 2013; Connelly et al, 2015)。栖息 地碎片化直接威胁多种传粉昆虫, 破坏了生态系统 中传粉网络的结构(Grass et al, 2018), 其中大部分 蜜蜂群体受损明显(Winfree et al, 2007)。本文讨论的 蜜蜂指蜜蜂总科所有蜂种, 它们是主要的膜翅目传 粉昆虫, 约有两万种, 其中社会性蜜蜂少于 1,000 种, 全球分布广泛。

景观中的开花植物种类直接决定了蜜蜂的食 物和营养组成。Donkersley等(2014)发现景观生态组 成直接影响蜂粮营养组成, 草地和阔叶林占比越高, 蜂粮的蛋白含量越高, 而蛋白质和氨基酸被认为是 决定蜜蜂营养的关键因素(Di Pasquale et al, 2016; Glavinic et al, 2017)。也有研究认为景观组成对蜂粮 没有决定性影响(Danner et al, 2017), 不过这些研究 只关注了景观组成多样性的高低, 并未考虑不同景 观生态系统中开花植物的种类和组成特点。景观生 态常常被划分为不同类别, 比如农田、森林、草地 等, 或者被归类于不同性质, 比如根据多样性的高 低分为简单和复杂, 或者根据人为影响程度分为农 田、自然和半自然。因此, 同一类性质的景观内植 被组成可能非常不同。Colwell等(2017)调查了不同 农田系统中西方蜜蜂(Apis mellifera)采集的蜂花粉 的多样性和营养状况, 发现不同农田系统中蜜蜂的 取食多样性和蜂粮蛋白含量均有显著差异。农业生 产影响传粉蜜蜂的另一主要因素是农药残留(Potts et al, 2010; Gill et al, 2012; Botías et al, 2015; Rundlöf et al, 2015; Crall et al, 2018), 它很可能是造成西方 蜜蜂蜂群崩溃综合征(colony collapse disorder, CCD) 的原因之一(Oldroyd, 2007; Goulson et al, 2015; Steinhauer et al, 2018)。

虽然与景观环境相关的因素明显影响着蜜蜂, 但其作用机制尚不明确。越来越多的研究显示肠道 菌群与宿主健康紧密相关, 早在2007年就有研究表 明患有崩溃综合征的蜂群其肠道菌群与正常蜂群 不一样, 且与一种病毒有密切关系(Cox-Foster et al, 2007)。Ribière等(2018)发现健康蜂群和不健康蜂群 的肠道微生物有显著差异。肠道有益菌可抵抗疾病, 促进宿主健康, 比如熊蜂肠道中的很多菌株都可以 抵抗病原菌(Praet et al, 2018)。相反, 菌群紊乱会降 低宿主对病原菌的抵抗力(Schwarz et al, 2016; Li et al, 2017a), 不利于蜜蜂健康(Anderson \& Ricigliano, 2017)。因此, 通过肠道微生物的视角可以帮助我们 理解景观环境影响蜜蜂健康的机制。

\section{景观差异与蜜蜂肠道菌群差异相关}

目前已有少量研究从景观水平上调查蜜蜂的 肠道微生物。Jones等(2018)通过比较16S rRNA V4 区序列发现, 景观生态对西方蜜蜂肠道微生物有显 著影响, 虽然油菜花田这一景观因素对菌群差异的 解释量只有 $6 \%$, 低于取样点造成的差异 $(20 \%)$, 但 如果综合考虑其他景观因素如开花植物的多样性 组成、农田和森林等的组成比例、农药残留等, 可 能会发现景观环境与肠道菌群演替有更显著的相 关性。为了探究同一种东方蜜蜂(Apis cerana)在不 同景观生态环境中的肠道菌群是否有差异, 我们分 别从不同蜂场取工蜂并测序其肠道中16S rRNA V3 区片段多样性(未发表数据)。4个蜂场分别位于四川 关坝(SCGB)、四川小河沟自然保护区(SCXHG)、陕 西杉树坪(SXSSP)和陕西杨家沟(SXYJG)(海拔分别 为 $1,333 \mathrm{~m} 、 1,410 \mathrm{~m} 、 1,484 \mathrm{~m}$ 和1,348 $\mathrm{m}$ )。关坝与小 河沟地区的植被组成相似, 后者划入自然保护区时 间长, 自然修复更好; 关坝和杨家沟蜂场附近有村 庄和农田, 而且据当地调查, 农田有施农药的情况; 
杉树坪和杨家沟同属秦岭自然保护区，前者自然修 复更好, 附近无人居住。所有样品工蜂属于同一东 方蜜蜂群体(Chen et al, 2018), 四川与陕西的工蜂 肠道菌群并无显著差异(图1a), 但来自同一省份的 工蜂之间肠道菌群差异显著小于来自不同省份的 (图1b), 这很可能与植被组成不同有关, 需要后期 更多的数据验证。关坝与杨家沟采样点附近均有村 庄, 而小河沟与杉树坪采样点的自然修复程度更高, 因此我们预期, 关坝与杨家沟所采蜜蜂的肠道菌群 组成差异应该更小, 而小河沟与杨家沟所采蜜蜂的 肠道菌群组成差异更大。但结果并不支持该假设, 暗示了研究土地利用对肠道菌群影响的实验设计 应该限定在更小的范围内, 例如未来可以在同一省 份植被组成相同或相似的地方设点采样。未来的工 作中我们将验证是否是景观环境造成了这些差异, 并研究其中哪些景观因素起决定性作用, 其作用机 制如何。

\section{2 花粉和农药对蜜蜂肠道菌群的影响}

花粉作为蜜蜂的主要食物来源, 对蜜蜂健康非 常重要(Frias et al, 2016), 营养压力也被认为是导致 CCD的原因之一(Naug, 2009; Potts et al, 2010; Goulson et al, 2015)。蜜蜂面临的另一主要生存压力是 农药残留。花粉营养物质和农药残留对蜜蜂的影响

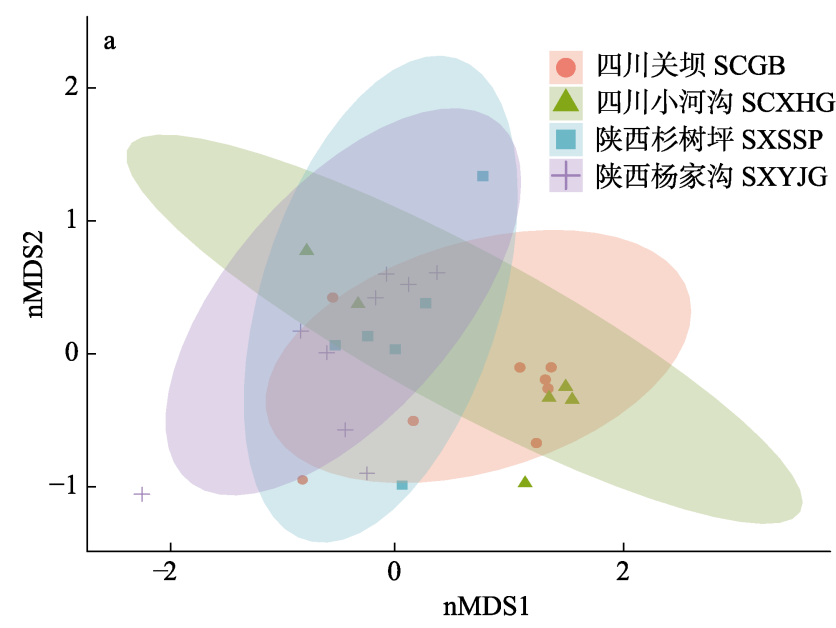

以及机制有待深入研究。

\section{1 花粉营养显著影响蜜蜂及其肠道微生物}

我们可以通过了解景观中蜜蜂获取的食物对 其肠道菌及其代谢物的影响来理解不同景观对蜜 蜂的影响。DeGrandi-Hoffman等(2016)研究发现相 比人工配方喂养, 天然饲养的西方蜜蜂更少染病, 而且越冬存活率更高。Di Pasquale等(2016)的研究 结果显示, 食物中花粉比例越高, 西方蜜蜂存活率 越高。有研究探究了花粉的营养成分和多样性对蜜 蜂健康的影响。Di Pasquale等(2013)给西方蜜蜂哺 育蜂喂食单种或等量混合的4种不同花粉(对照组只 喂食糖水), 并监测蜜蜂生理特征及其对病原菌 Nosema ceranae的抵抗力, 发现不同的花粉对哺育 蜂的发育和对病原菌抵抗力的影响存在显著差异: 氨基酸含量高的花粉更有利于哺育蜂抵抗病原菌, 而混合花粉未表现出优势。但在被N. ceranae感染后, 喂食混合花粉或蛋白质含量最高的花粉的蜜蜂存 活期更长。除蛋白质外, 花粉还提供蜜蜂所需的脂 类和微量元素, 花蜜提供糖类物质, 因此景观中的 开花植物种类直接影响蜜蜂健康 (Vaudo et al, 2015)。

大量研究表明食物(营养)是影响肠道微生物的 重要因素(Muegge et al, 2011; Wu et al, 2011; David et al, 2014; Li et al, 2017b)。蜜蜂肠道菌群在宿主消

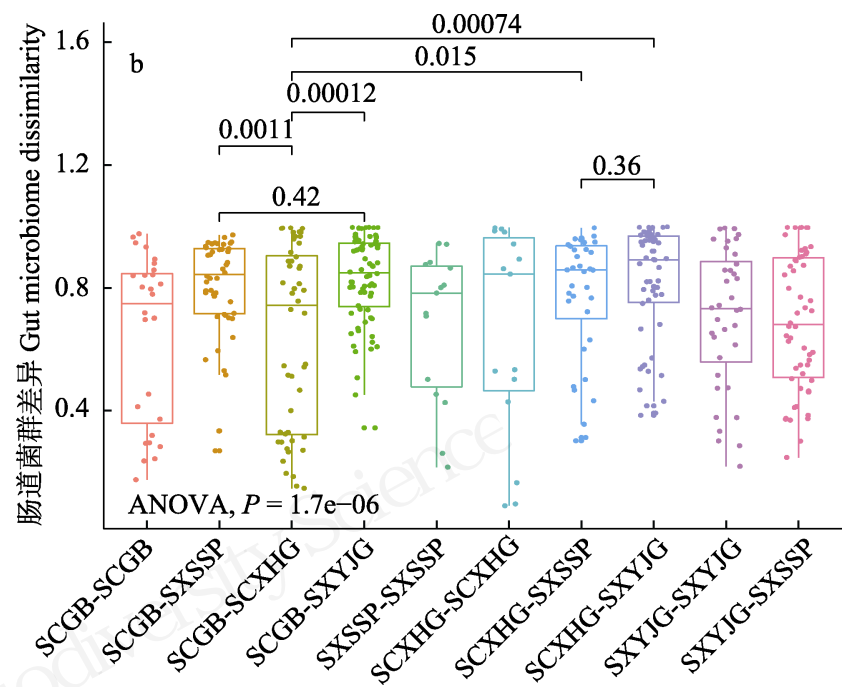

图1 来自 4 个蜂场的东方蜜蜂肠道菌群16S rRNA V3 片段序列的非度量多维尺度分析(nMDS)图(a)及蜂场内和蜂场间的 Bray-Curtis距离比较(ANOVA和t检验, b)

Fig. 1 Gut microbiome similarity on 16S rRNA V3 region of Apis cerana sampled from 4 apiaries showed by non-metric multidimensional scaling (nMDS) plot (a), and Bray-Curtis distance between gut microbiome compared among and between sampling sites and tested by using ANOVA and $t$-test (b). SCGB, Guanba, Sichuan; SCXHG, Xiaohegou, Sichuan; SXSSP, Shanshuping, Shaanxi; SXYJG, Yangjiagou, Shaanxi. 
化食物、吸收营养的过程中扮演着关键的角色 (Engel \& Bonilla-Rosso, 2018)。例如西方蜜蜂肠道 菌可以消化很多种类的粉源营养物质, 更重要的是 可以降解难以利用的植物次级代谢产物和花粉壁 (Kešnerová et al, 2017), 其肠道菌Gilliamella apicola 的一些菌株还可以降解对蜜蜂有毒的糖类(Zheng et al, 2016)。食物可以通过影响肠道微生物进而作用 于宿主。Maes等(2016)发现喂食陈旧的花粉可显著 影响西方蜜蜂肠道微生物组成, 并导致死亡率升高 和生长发育缺陷, 而且喂食花粉替代品会加重这些 不良影响。

\section{2 农药残留严重损害蜜蜂并显著改变蜜蜂肠 道微生物}

Pettis等(2013)从西方蜜蜂蜂粮中检测出35种残 留农药, 发现农药可削弱西方蜜蜂对病原菌 Nosema ceranae的抵抗力, 而且Kessler等(2015)研 究发现西方蜜蜂不会趋避新烟碱类农药。大量研究 表明: 个体水平上, 农药显著降低工蜂存活率 (Henry et al, 2012; Tsvetkov et al, 2017)及蜂王的繁 殖力和新蜂王产量(Whitehorn et al, 2012; Tsvetkov et al, 2017; Siviter et al, 2018); 蜂群水平上, 新烟碱 农药被确证对西方蜜蜂、熊蜂和野生蜂蜂群不利: 蜂群增长量显著降低, 后代明显减少, 蜂王产出率 也降低了 85\% (Whitehorn et al, 2012; Woodcock et al, 2017); 还有一些研究发现农药在免疫(Tsvetkov et $\mathrm{al}, 2017$ )、发育(dos Santos et al, 2016)和行为(Henry et al, 2012)水平上对蜜蜂造成损伤。

另一方面, 人们也意识到自然栖息地生态组成 对传粉昆虫多样性的重要性。在农作系统中, 人为 增加生态多样性有利于缓解传粉生态功能缺失的 状况(Blaauw \& Isaacs, 2014a, b; Kovács-Hostyánszki et al, 2017)。在Colwell等(2017)研究的不同农作系统 中, 农药残留与开花植物的多样性呈负相关。生态 强化过程中, 可能是农药被 “稀释”了 (Park et al, 2015; Colwell et al, 2017), 也可能是蜜蜂因为景观 环境的改善变得更健康了。

已有少量研究从肠道微生物的角度解析农药 影响蜜蜂的作用机制。Kakumanu等(2016)实验发现 暴露于蝇毒磷、氟胺氰菊酯和百菌清的西方蜜蜂的 肠道微生物发生显著改变。Yang等(2019)发现不同 农药对东方蜜蜂和西方蜜蜂的中肠菌群的影响不 同, 科学家正致力于从机理层面解释这种影响。比
如, 除草剂草甘膦的作用靶标物为烯醇式丙酮基莽 草-3-磷酸合成酶, 该酶存在于一些植物和微生物中, 也存在于几乎所有已测序的西方蜜蜂肠道菌中。 Motta等(2018)发现蜜蜂肠道菌编码的这种酶有两 种类型，一种对草甘膦敏感，另一种不敏感，另外 还有些菌株可以通过未知的方式抵抗草甘膦。这些 基础研究可以帮助我们理解农药作用于蜜蜂和蜜 蜂抵抗农药的机制。

\section{景观花源微生物对蜜蜂肠道菌群的影响}

除了食物和农药, 景观环境中还有另外一个重 要因素可扰动蜜蜂肠道微生物, 即花源微生物。Lim 等(2015)将景观看作一个整体变量, 发现多种熊蜂 的肠道微生物组成都与之相关, 因此他们认为至少 部分肠道菌是可以通过环境传播的。还有研究表明 景观影响蜂巢和蜂粮微生物。Donkersley等(2018) 发现土地利用会影响蜂粮菌群的组成, 通过将多种 景观进行比较, 发现一种改善后的草地系统中的蜂 粮菌群多样性最低, 而蜂粮微生物可直接作用于蜜 蜂肠道微生物(Powell et al, 2014)。景观生态的变化 会显著改变花粉和花蜜资源(Baude et al, 2016), 与 之相应的花源微生物不可忽视。

\section{1 花源微生物可能是肠道微生物的来源之一}

荀二娜等(2017)总结了花蜜微生物的相关研究, 发现每种花蜜所含细菌虽然平均只有 1.4 种, 但发 生率较高。这些细菌可以适应花蜜中的高糖环境, 属于一类独特的微生物, 一般为变形菌门, 而且通 常具有花蜜植物特异性(Fridman et al, 2012)。目前 研究较多的西方蜜蜂肠道的共生菌种类不多, 其中 很多都属于变形菌(Martinson et al, 2011)。Anderson 等(2013)培养和检测出传粉环境中的花蜜与西方蜜 蜂肠道共有的多种细菌。目前蜜蜂肠道菌是否来源 于环境还没有定论。研究较多的社会性蜜蜂——西 方蜜蜂、东方蜜蜂和熊蜂拥有简单而稳定的肠道菌 群(Martinson et al, 2011), 而且与宿主健康息息相关 (Koch \& Schmid-Hempel, 2011; Anderson \& Ricigliano, 2017; Raymann \& Moran, 2018), 其核心菌 (Martinson et al, 2011; Kwong et al, 2017)未在其他 环境中被发现, 社会性行为很可能有助于这些蜜蜂 肠道菌群的传播和稳定(Kwong et al, 2017)。不过, 绝大多数研究都是采用 $16 \mathrm{~S}$ rRNA扩增子片段作为 分析手段, 其中核心菌占比高达 $90 \%$ 以上, 非核心 
菌容易被忽略。Saraiva等(2015)发现蜂花粉中微生 物与西方蜜蜂肠道微生物组成存在显著差异, 但依 然共享了 7\%的细菌物种。从Anderson等(2014)研究 西方蜜蜂蜂花粉的微生物结果可以看出, 西方蜜蜂 肠道的常见优势菌可以较长时间(至少 $3 \mathrm{~d}$ )存活于花 粉环境, 可满足菌群水平传播的必要条件。因此, 这类拥有稳定菌群的社会性蜜蜂有可能获得并保 留环境微生物, 这些环境菌对蜜蜂及其肠道菌群的 作用有待研究。

野生蜜蜂的肠道菌群与东方蜜蜂、西方蜜蜂和 熊蜂显著不同, 也与花粉明显相关, 但共同点是它 们都“招募”了对自身有益的细菌(Graystock et al, 2017)。McFrederick等(2012, 2014, 2017)发现多种野 生蜂的肠道与花共享细菌, 他们认为蜜蜂很可能从 花粉和花蜜中获得了微生物。McFrederick和Rehan (2016)分析了一种木蜂巢内花粉和细菌的多样性, 虽然总体上没有发现花粉多样性与细菌多样性的 相关性, 但其中某些花粉与对应的优势细菌显著相 关, 这提示花粉可能是细菌的来源。绝大多数野生 传粉蜜蜂是独居蜂, 没有社会性行为帮助它们保持 稳定的肠道微生物, 尽管对其肠道菌群的研究较少, 但推测环境微生物可能对其有更显著的影响。

\section{2 花源微生物对不同蜜蜂肠道菌的影响可能 不同}

Zou等(2017)研究了农田占比10\%-70\%的不同 景观系统对传粉昆虫多样性的影响, 发现农田比例 越高, 野生蜂的多样性越低, 其中独居蜂受影响显 著, 而西方蜜蜂的丰度并未受到显著的影响。另外 有研究表明, 不同的野生蜂多样性与植被种类有关 (Rollin et al, 2013; Wu et al, 2018), 说明不同的蜜蜂 受景观的影响不同, 野生独居蜂比社会性蜜蜂更容 易受到景观生态组成变化的影响(Neokosmidis et al, 2018)。尽管人工养殖的蜜蜂对农作物生产和自然生 态系统传粉功能贡献显著 (Hung et al, 2018; Saunders et al, 2018), CCD也让西方蜜蜂备受关注, 但传粉者的多样性而非数量决定了传粉功能的水 平(Winfree et al, 2015; Blitzer et al, 2016; Kremen, 2018), 且野生蜂对传粉网络和传粉服务的贡献不 亚于饲养蜜蜂(Brittain et al, 2012; Holzschuh et al, 2012; Garibaldi et al, 2014)。过多地饲养蜜蜂可能会 破坏自然的传粉网络结构并威胁野生传粉者 (Torné-noguera et al, 2016, Magrach et al, 2017;
Geldmann \& Gonzalez-Varo, 2018)。有研究表明野生 熊蜂受制于养殖蜜蜂，但这种影响会因为景观生态 的多样性升高而有所改善(Herbertsson et al, 2016)。 由此可见景观环境对不同蜜蜂的发展十分重要。

鉴于各种蜜蜂肠道菌群的差异, 研究景观环境 对其的影响需要进行大量工作，蜜蜂肠道菌联盟 (BeeBiome Consortium)也强调了这一研究方向的重 要性(Engel et al, 2016)。景观生态的改变意味着环 境微生物“菌池”的变化，这是一把双刃剑：既可能 提供有益菌(Graystock et al, 2017; McFrederick et al, 2017)，又可能隐藏致病菌 (Graystock et al，2015; Rothman et al, 2018)。最近发表的一项研究(Keller et al，2018)表明，从巢穴环境分离培养出来的普遍存 在于一种独居蜂体表、肠道和巢穴的一株细菌可有 效抑制致病真菌和细菌。因此探明环境微生物影响 肠道微生物进而作用于蜜蜂的机制和规律能为我 们保护蜜蜂提供新的视角, 改变蜜蜂肠道微生物可 能是一种有效促进蜜蜂健康的干预方法(Crotti et al, 2013)。

综上所述, 景观环境所决定的蜜蜂食物组成、 花源微生物和农药都可能显著影响蜜蜂, 其影响机 制可以从肠道微生物的角度探究(图2)。在以上所述 的景观环境差异影响蜜蜂的研究基础上，需要加入 不同处理组与对照组的菌群比较分析，从差异显著 的菌种、菌株、基因及其调控和代谢产物等方面验

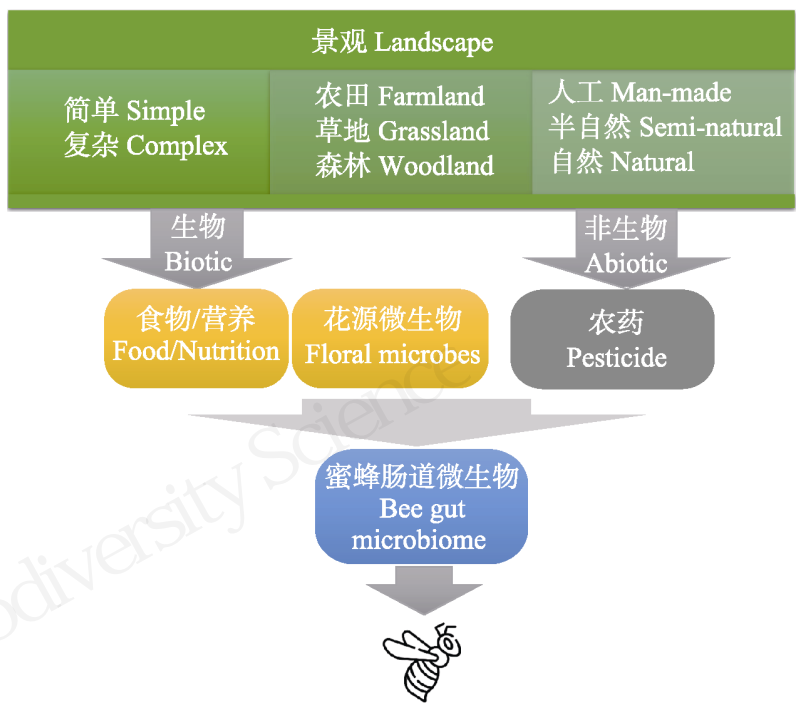

图2 从肠道微生物角度探究景观环境可能影响蜜蜂的主要 途径示意图

Fig. 2 The main possible paths through which the landscape could affect bees by changing their microbiome 
证景观变化扰动蜜蜂肠道微生物, 进而影响蜜蜂健 康和蜂群发展的假设, 有望用蜜蜂肠道菌群指示景 观组成是否生态友好。近期研究发现, 自然修复工 作的联通栖息地和强化生态组成均有利于物种多 样性的升高和传粉网络的稳固 (Blaauw \& Isaacs, 2014a, b; Aizen et al, 2016; Kovács-Hostyánszki et al, 2017; Kleijn et al, 2018), 通过确定其中蜜蜂肠道微 生物的作用, 有望更合理高效地实施自然生态修复 工作。

致谢：感谢陕西省洋县长青林业局向定乾和四川省 平武县关坝村李芯锐帮助收集工蜂样品，感谢首都 师范大学顾垒老师对植物多样性调查工作的指导。

\section{参考文献}

Aizen MA, Gleiser G, Sabatino M, Gilarranz LJ, Bascompte J, Verdú M (2016) The phylogenetic structure of plantpollinator networks increases with habitat size and isolation. Ecology Letters, 19, 29-36.

Anderson KE, Ricigliano VA (2017) Honey bee gut dysbiosis: A novel context of disease ecology. Current Opinion in Insect Science, 22, 125-132.

Anderson KE, Carroll MJ, Sheehan T, Mott BM, Maes P, Corby-Harris V (2014) Hive-stored pollen of honey bees: Many lines of evidence are consistent with pollen preservation, not nutrient conversion. Molecular Ecology, 23, 5904-5917.

Anderson KE, Sheehan TH, Mott BM, Maes P, Snyder L, Schwan MR, Walton A, Jones BM, Corby-Harris V (2013) Microbial ecology of the hive and pollination landscape: Bacterial associates from floral nectar, the alimentary tract and stored food of honey bees (Apis mellifera). PLoS ONE, 8, e83125.

Baude M, Kunin WE, Boatman ND, Conyers S, Davies N, Gillespie MAK, Morton RD, Smart SM, Memmott J (2016) Historical nectar assessment reveals the fall and rise of floral resources in Britain. Nature, 530, 85-88.

Biesmeijer JC, Roberts SPM, Reemer M, Ohlemüller R, Edwards M, Peeters T, Schaffers AP, Potts SG, Kleukers R, Thomas CD, Settele J, Kunin WE (2006) Parallel declines in pollinators and insect-pollinated plants in Britain and the Netherlands. Science, 313, 351-355.

Blaauw BR, Isaacs R (2014a) Flower plantings increase wild bee abundance and the pollination services provided to a pollination-dependent crop. Journal of Applied Ecology, 51, 890-898.

Blaauw BR, Isaacs R (2014b) Larger patches of diverse floral resources increase insect pollinator density, diversity, and their pollination of native wild flowers. Basic and Applied Ecology, 15, 701-711.

Blitzer EJ, Gibbs J, Park MG, Danforth BN (2016) Pollination services for apple are dependent on diverse wild bee communities. Agriculture, Ecosystems and Environment, 221, 1-7.

Botías C, David A, Horwood J, Abdul-Sada A, Nicholls E, Hill E, Goulson D (2015) Neonicotinoid residues in wildflowers, a potential route of chronic exposure for bees. Environmental Science Technology, 49, 12731-12740.

Brittain C, Williams N, Kremen C, Klein AM (2012) Synergistic effects of non-Apis bees and honey bees for pollination services. Proceedings of the Royal Society B: Biological Sciences, 280, 20122767.

Burkle LA, Marlin JC, Knight TM (2013) Plant-pollinator interactions over 120 years: Loss of species, co-occurrence, and function. Science, 339, 1611-1616.

Chen C, Wang H, Liu Z, Chen X, Tang J, Meng F, Shi W (2018) Population genomics provide insights into the evolution and adaptation of the eastern honey bee (Apis cerana). Molecular Biology and Evolution, 35, 2260-2271.

Colwell MJ, Williams GR, Evans RC, Shutler D (2017) Honey bee-collected pollen in agro-ecosystems reveals diet diversity, diet quality, and pesticide exposure. Ecology and Evolution, 7, 7243-7253.

Connelly H, Poveda K, Loeb G (2015) Landscape simplification decreases wild bee pollination services to strawberry. Agriculture, Ecosystems and Environment, 211, 51-56.

Cox-Foster DL, Conlan S, Holmes EC, Palacios G, Evans JD, Moran NA, Quan PL, Briese T, Horning M, Geiser DM, Martinson V, vanEngelsdorp D, Kalkstein AL, Drysdale A, Hui J, Zhai J, Cui L, Hutchison SK, Simons JF, Egholm M, Pettis JS, Lipkin WI (2007) A metagenomic survey of microbes in honey bee colony collapse disorder. Science, 318, 283-288.

Crall JD, Switzer CM, Oppenheimer RL, Ford Versypt AN, Dey B, Brown A, Eyster M, Guerin C, Pierce NE, Combes SA, de Bivort BL (2018) Neonicotinoid exposure disrupts bumblebee nest behavior, social networks, and thermoregulation. Science, 362, 683-686.

Crotti E, Sansonno L, Prosdocimi EM, Vacchini V, Hamdi C, Cherif A, Conella E, Marzorati M, Balloi A (2013) Microbial symbionts of honeybees: A promising tool to improve honeybee health. New Biotechnology, 30, 716-722.

Danner N, Keller A, Härtel S, Steffan-Dewenter I (2017) Honey bee foraging ecology: Season but not landscape diversity shapes the amount and diversity of collected pollen. PLoS ONE, 12, e0183716.

David LA, Maurice CF, Carmody RN, Gootenberg DB, Button JE, Wolfe BE, Ling AV, Devlin AS, Varma Y, Fischbach MA, Biddinger SB, Dutton RJ, Turnbaugh PJ (2014) Diet rapidly and reproducibly alters the human gut microbiome. 
Nature, 505, 559-563.

DeGrandi-Hoffman G, Chen Y, Rivera R, Carroll M, Chambers M, Hidalgo G, de Jong EW (2016) Honey bee colonies provided with natural forage have lower pathogen loads and higher overwinter survival than those fed protein supplements. Apidologie, 47, 186-196.

Di Pasquale G, Alaux C, Le Conte Y, Odoux JF, Pioz M, Vaissière BE, Belzunces LP, Decourtye A (2016) Variations in the availability of pollen resources affect honey bee health. PLoS ONE, 11, e0162818.

Di Pasquale G, Salignon M, Le Conte Y, Belzunces LP, Decourtye A, Kretzschmar A, Suchail S, Brunet JL, Alaux C (2013) Influence of pollen nutrition on honey bee health: Do pollen quality and diversity matter? PLoS ONE, 8, e72016.

Donkersley P, Rhodes G, Pickup RW, Jones KC (2018) Bacterial communities associated with honeybee food stores are correlated with land use. Ecology and Evolution, 8, 4743-4756.

Donkersley P, Rhodes G, Pickup RW, Jones KC, Wilson K (2014) Honeybee nutrition is linked to landscape composition. Ecology and Evolution, 4, 4195-4206.

dos Santos CF, Acosta AL, Dorneles AL, dos Santos PDS, Blochtein B (2016) Queens become workers: Pesticides alter caste differentiation in bees. Scientific Reports, 6, 31605.

Engel P, Bonilla-Rosso G (2018) Functional roles and metabolic niches in the honey bee gut microbiota. Current Opinion in Microbiology, 43, 69-76.

Engel P, Kwong WK, McFrederick Q, Anderson KE, Barribeau SM, Chandler JA, Cornman RS, Dainat J, de Miranda JR, Doublet V, Emery O, Evans JD, Farinelli L, Flenniken ML, Granberg F, Grasis JA, Gauthier L, Hayer J, Koch H, Kocher S, Martinson VG, Moran N, Munoz-Torres M, Newton I, Paxton RJ, Powell E, Sadd BM, Schmid-Hempel P, Schmid-Hempel R, Song SJ, Schwarz RS, vanEngelsdorp D, Dainat B (2016) The bee microbiome: Impact on bee health and model for evolution and ecology of host-microbe interactions. mBio, 7, e02164-15.

Frias BED, Barbosa CD, Lourenço AP (2016) Pollen nutrition in honey bees (Apis mellifera): Impact on adult health. Apidologie, 47, 15-25.

Fridman S, Izhaki I, Gerchman Y, Halpern M (2012) Bacterial communities in floral nectar. Environmental Microbiology Reports, 4, 97-104.

Garibaldi LA, Steffan-dewenter I, Winfree R, Aizen MA, Bommarco R, Cunningham SA, Kremen C, Carvalheiro LG, Harder LD, Afik O, Bartomeus I, Benjamin F, Boreux V, Cariveau D, Chacoff NP, Dudenhöffer JH, Freitas BM, Ghazoul J, Greenleaf S, Hipólito J, Holzschuh A, Howlett B, Isaacs R, Javorek SK, Kennedy CM, Krewenka KM, Krishnan S, Mandelik Y, Mayfield MM, Motzke I, Munyuli T, Nault BA, Otieno M, Petersen J, Pisanty G, Potts SG, Rader R, Ricketts TH, Rundlöf M, Seymour CL, Schüepp C, Szentgyörgyi H, Taki H, Tscharntke T, Vergara CH, Viana
BF, Wanger TC, Westphal C, Williams N, Klein AM (2014) Wild pollinators enhance fruit set of crops regardless of honey bee abundance. Science, 339, 1608-1611.

Geldmann J, Gonzalez-Varo JP (2018) Conserving honey bees does not help wildlife: High densities of managed honey bees can harm populations of wild pollinators. Science, 359, 392-393.

Gill RJ, Baldock KCR, Brown MJF, Cresswell JE, Dicks LV, Fountain MT, Garratt MPD, Gough LA, Heard MS, Holland JM, Ollerton J, Stone GN, Tang CQ, Vanbergen AJ, Vogler A, Woodward G, Arce AN, Boatman ND, Brand-Hardy R, Breeze TD, Green M, Hartfield CM, O’Connor RS, Osborne JL, Phillips J, Sutton PB, Potts SG (2016) Protecting an ecosystem service: Approaches to understanding and mitigating threats to wild insect pollinators. Advances in Ecological Research, 54, 135-206.

Gill RJ, Ramos-Rodriguez O, Raine NE (2012) Combined pesticide exposure severely affects individual- and colonylevel traits in bees. Nature, 491, 105-108.

Glavinic U, Stankovic B, Draskovic V, Stevanovic J, Petrovic T, Lakic N, Stanimirovic Z (2017) Dietary amino acid and vitamin complex protects honey bee from immunosuppression caused by Nosema ceranae. PLoS ONE, 12, e0187726.

Goulson D, Nicholls E, Botías C, Rotheray EL (2015) Bee declines driven by combined stress from parasites, pesticides, and lack of flowers. Science, 347, 1255957.

Grass I, Jauker B, Steffan-Dewenter I, Tscharntke T, Jauker F (2018) Past and potential future effects of habitat fragmentation on structure and stability of plant-pollinator and host-parasitoid networks. Nature Ecology \& Evolution, 2, 1408-1417.

Graystock P, Goulson D, Hughes WOH (2015) Parasites in bloom: Flowers aid dispersal and transmission of pollinator parasites within and between bee species. Proceedings of the Royal Society B: Biological Sciences, 282, https://doi.org/10.1098/rspb.2015.1371.

Graystock P, Rehan SM, McFrederick QS (2017) Hunting for healthy microbiomes: Determining the core microbiomes of Ceratina, Megalopta, and Apis bees and how they associate with microbes in bee collected pollen. Conservation Genetics, 18, 701-711.

Henry M, Béguin M, Requier F, Rollin O, Odoux J, Aupinel P, Tchamitchian S, Decourtye A (2012) A common pesticide decreases foraging success and survival in honey bees. Science, 336, 348-350.

Herbertsson L, Lindström SAM, Rundlöf M, Bommarco R, Smith HG (2016) Competition between managed honeybees and wild bumblebees depends on landscape context. Basic and Applied Ecology, 17, 609-616.

Holzschuh A, Dudenhöffer J, Tscharntke T (2012) Landscapes with wild bee habitats enhance pollination, fruit set and yield of sweet cherry. Biological Conservation, 153, 101-107. 
Hung KLJ, Kingston JM, Albretch M, Holway DA, Kohn JR (2018) The worldwide importance of honey bees as pollinators in natural habitats. Proceedings of the Royal Society B: Biological Sciences, 285, 20172140.

Jones JC, Fruciano C, Hildebrand F, Al Toufalilia H, Balfour NJ, Bork P, Engel P, Ratnieks FL, Hughes WO (2018) Gut microbiota composition is associated with environmental landscape in honey bees. Ecology and Evolution, 8, 441-451.

Kakumanu ML, Reeves AM, Anderson TD, Rodrigues RR, Williams MA (2016) Honey bee gut microbiome is altered by in-hive pesticide exposures. Frontiers in Microbiology, 7, $1-11$.

Keller A, Brandel A, Becker MC, Balles R, Abdelmohsen UR, Ankenbrand MJ, Sickel W (2018) Wild bees and their nests host Paenibacillus bacteria with functional potential of avail. Microbiome, 6, 1-10.

Kešnerová L, Mars RAT, Ellegaard KM, Troilo M, Sauer U, Engel P (2017) Disentangling metabolic functions of bacteria in the honey bee gut. PLoS Biology, 15, e2003467.

Kessler SC, Tiedeken EJ, Simcock KL, Derveau S, Mitchell J, Softley S, Radcliffe A, Stout JC, Wright GA (2015) Bees prefer foods containing neonicotinoid pesticides. Nature, 521, 74-76.

Kleijn D, Bommarco R, Fijen TPM, Garibaldi LA, Potts SG, van der Putten WH (2018) Ecological intensification: Bridging the gap between science and practice. Trends in Ecology and Evolution, 34, 154-166.

Klein A, Vaissie BE, Steffan-dewenter I, Cunningham SA, Kremen C, Cedex A (2007) Importance of pollinators in changing landscapes for world crops. Proceedings of the Royal Society B: Biological Sciences, 274, 303-313.

Koch H, Schmid-Hempel P (2011) Socially transmitted gut microbiota protect bumble bees against an intestinal parasite. Proceedings of the National Academy of Sciences, USA, 108, 19288-19292.

Kovács-Hostyánszki A, Espíndola A, Vanbergen AJ, Settele J, Kremen C, Dicks LV (2017) Ecological intensification to mitigate impacts of conventional intensive land use on pollinators and pollination. Ecology Letters, 20, 673-689.

Kremen C (2018) The value of pollinator species diversity. Science, 359, 741-742.

Kremen C, Williams NM, Aizen MA, Gemmill-Herren B, Lebuhn G, Minckley R, Packer L, Potts SG, Roulston T, Steffan-Dewenter I, Vázquez DP, Winfree R, Adams L, Crone EE, Greenleaf SS, Keitt TH, Klein AM, Regetz J, Ricketts TH (2007) Pollination and other ecosystem services produced by mobile organisms: A conceptual framework for the effects of land-use change. Ecology Letters, 10, 299-314.

Kwong WK, Medina LA, Koch H, Sing KW, Soh EJY, Ascher JS, Jaffe R, Moran NA (2017) Dynamic microbiome evolution in social bees. Science Advances, 3, e1600513.
Li JH, Evans JD, Li WF, Zhao YZ, Degrandi-hoffman G, Huang SK, Li ZG, Hamilton M, Chen YP (2017a) New evidence showing that the destruction of gut bacteria by antibiotic treatment could increase the honey bee's vulnerability to Nosema infection. PLoS ONE, 12, e0187505.

Li Q, Lauber CL, Czarnecki-Maulden G, Pan Y (2017b) Effects of the dietary protein and carbohydrate ratio on gut microbiomes in dogs of different body conditions. mBio, 8, e01703-16.

Lim HC, Chu CC, Seufferheld MJ, Cameron SA (2015) Deep sequencing and ecological characterization of gut microbial communities of diverse bumble bee species. PLoS ONE, 10, e0118566.

Maes PW, Rodrigues PAP, Oliver R, Mott BM, Anderson KE (2016) Diet-related gut bacterial dysbiosis correlates with impaired development, increased mortality and Nosema disease in the honeybee (Apis mellifera). Molecular Ecology, 25, 5439-5450.

Magrach A, González-Varo JP, Boiffier M, Vilà M, Bartomeus I (2017) Honeybee spillover reshuffles pollinator diets and affects plant reproductive success. Nature Ecology \& Evolution, 1, 1299-1307.

Martinson VG, Danforth BN, Minckley RL, Rueppell O, Tingek S, Moran NA (2011) A simple and distinctive microbiota associated with honey bees and bumble bees. Molecular Ecology, 20, 619-628.

McFrederick QS, Rehan SM (2016) Characterization of pollen and bacterial community composition in brood provisions of a small carpenter bee. Molecular Ecology, 25, 2302-2311.

McFrederick QS, Thomas JM, Neff JL, Vuong HQ, Russell KA, Hale AR, Mueller UG (2017) Flowers and wild megachilid bees share microbes. Microbial Ecology, 73, 188-200.

McFrederick QS, Wcislo WT, Taylor DR, Ishak HD, Dowd SE, Mueller UG (2012) Environment or kin: Whence do bees obtain acidophilic bacteria? Molecular Ecology, 21, 1754-1768.

McFrederick QS, Wcislo WT, Hout MC, Mueller UG (2014) Host species and developmental stage, but not host social structure, affects bacterial community structure in socially polymorphic bees. FEMS Microbiology Ecology, 88, 398-406.

Motta EVS, Raymann K, Moran NA (2018) Glyphosate perturbs the gut microbiota of honey bees. Proceedings of the National Academy of Sciences, USA, 115, 10305-10310.

Muegge BD, Kuczynski J, Knights D, Clemente JC, González A, Fontana L, Henrissat B, Knight R, Gordon JI (2011) Diet drives convergence in gut microbiome functions across mammalian phylogeny and within humans. Science, 332, 970-974.

Murphy GEP, Romanuk TN (2014) A meta-analysis of declines in local species richness from human disturbances. Ecology and Evolution, 4, 91-103.

Naug D (2009) Nutritional stress due to habitat loss may 
explain recent honeybee colony collapses. Biological Conservation, 142, 2369-2372.

Neokosmidis L, Tscheulin T, Devalez J, Petanidou T (2018) Landscape spatial configuration is a key driver of wild bee demographics. Insect Science, 25, 172-182.

Oldroyd BP (2007) What's killing American honey bees? PLoS Biology, 5, e168.

Ollerton J, Erenler H, Edwards M, Crockett R (2014) Extinctions of aculeate pollinators in Britain and the role of large-scale agricultural changes. Science, 346, 1360-1362.

Ollerton J, Winfree R, Tarrant S (2011) How many flowering plants are pollinated by animals? Oikos, 120, 321-326.

Park MG, Blitzer EJ, Gibbs J, Losey JE, Danforth BN (2015) Negative effects of pesticides on wild bee communities can be buffered by landscape context. Proceedings of the Royal Society B: Biological Sciences, 282, 20150299.

Pettis JS, Lichtenberg EM, Andree M, Stitzinger J, Rose R, vanEngelsdorp D (2013) Crop pollination exposes honey bees to pesticides which alters their susceptibility to the gut pathogen Nosema ceranae. PLoS ONE, 8, e70182.

Potts SG, Biesmeijer JC, Kremen C, Neumann P, Schweiger O, Kunin WE (2010) Global pollinator declines: Trends, impacts and drivers. Trends in Ecology and Evolution, 25, 345-353.

Powell JE, Martinson VG, Urban-Mead K, Moran NA (2014) Routes of acquisition of the gut microbiota of the honey bee Apis mellifera. Applied and Environmental Microbiology, 80, 7378-7387.

Praet J, Parmentier A, Schmid-Hempel R, Meeus I, Smagghe G, Vandamme P (2018) Large-scale cultivation of the bumblebee gut microbiota reveals an underestimated bacterial species diversity capable of pathogen inhibition. Environmental Microbiology, 20, 214-227.

Ramankutty N, Mehrabi Z, Waha K, Jarvis L, Kremen C, Herrero M, Rieseberg LH (2018) Trends in global agricultural land use: Implications for environmental health and food security. Annual Review of Plant Biology, 69, 789-815.

Raymann K, Moran NA (2018) The role of the gut microbiome in health and disease of adult honey bee workers. Current Opinion in Insect Science, 26, 97-104.

Ribière C, Hegarty C, Stephenson H, Whelan P, O'Toole PW (2018) Gut and whole-body microbiota of the honey bee separate thriving and non-thriving hives. Microbial Ecology, 78, 195-205.

Rollin O, Bretagnolle V, Decourtye A, Aptel J, Michel N, Vaissière BE, Henry M (2013) Differences of floral resource use between honey bees and wild bees in an intensive farming system. Agriculture, Ecosystems and Environment, 179, 78-86.

Rothman JA, Andrikopoulos C, Cox-Foster D, McFrederick QS (2018) Floral and foliar source affect the bee nest microbial community. Microbial Ecology, https://doi.org/10. 1007/s00248-018-1300-3.
Rundlöf M, Andersson GKS, Bommarco R, Fries I, Hederström V, Herbertsson L, Jonsson O, Klatt BK, Pedersen TR, Yourstone J, Smith HG (2015) Seed coating with a neonicotinoid insecticide negatively affects wild bees. Nature, 521, 77-80.

Saraiva MA, Zemolin APP, Franco JL, Boldo JT, Stefenon VM, Triplett EW, de Oliveira Camargo FA, Roesch LFW (2015) Relationship between honeybee nutrition and their microbial communities. Antonie van Leeuwenhoek, 107, 921-933.

Saunders ME, Smith TJ, Rader R (2018) Bee conservation: Key role of managed bees. Science, 360, 389.

Schwarz RS, Moran NA, Evans JD (2016) Early gut colonizers shape parasite susceptibility and microbiota composition in honey bee workers. Proceedings of the National Academy of Sciences, USA, 113, 9345-9350.

Siviter H, Brown MJF, Leadbeater E (2018) Sulfoxaflor exposure reduces bumblebee reproductive success. Nature, 561, 109-112.

Steinhauer N, Kulhanek K, Antúnez K, Human H, Chantawannakul P, Chauzat MP, vanEngelsdorp D (2018) Drivers of colony losses. Current Opinion in Insect Science, 26, 142-148.

Torné-noguera A, Rodrigo A, Osorio S, Bosch J (2016) Collateral effects of beekeeping: Impacts on pollen-nectar resources and wild bee communities. Basic and Applied Ecology, 17, 199-209.

Tsvetkov N, Samson-Robert O, Sood K, Patel HS, Malena DA, Gajiwala PH, Maciukiewicz P, Fournier V, Zayed A (2017) Chronic exposure to neonicotinoids reduces honey bee health near corn crops. Science, 356, 1395-1397.

Vaudo AD, Tooker JF, Grozinger CM, Patch HM (2015) Bee nutrition and floral resource restoration. Current Opinion in Insect Science, 10, 133-141.

Whitehorn PR, O’Connor S, Wackers FL, Goulson D (2012) Neonicotinoid pesticide reduces bumble bee colony growth and queen production. Science, 336, 351-352.

Winfree R, Fox JW, Williams NM, Reilly JR, Cariveau DP (2015) Abundance of common species, not species richness, drives delivery of a real-world ecosystem service. Ecology Letters, 18, 626-635.

Winfree R, Griswold T, Kremen C (2007) Effect of human disturbance on bee communities in a forested ecosystem. Conservation Biology, 21, 213-223.

Woodcock BA, Bullock JM, Shore RF, Heard MS, Pereira MG, Redhead J, Ridding L, Dean H, Sleep D, Henrys P, Peyton J, Hulmes S, Hulmes L, Sárospataki M, Saure C, Edwards M, Genersch E, Knäbe S, Pywell RF (2017) Country-specific effects of neonicotinoid pesticides on honey bees and wild bees. Science, 356, 1393-1395.

Wu GD, Chen J, Hoffmann C, Bittinger K, Chen Y, Keilbaugh SA, Bewtra M, Knights D, Walters WA, Knight R, Sinha R, Gilroy E, Gupta K, Baldassano R, Nessel L, Li H, Bushman 
FD, Lewis JD (2011) Linking long-term dietary patterns with gut microbial enterotypes. Science, 334, 105-108.

Wu P, Axmacher JC, Song X, Zhang X, Xu H, Chen C, Yu Z, Liu Y (2018) Effects of plant diversity, vegetation composition, and habitat type on different functional trait groups of wild bees in rural Beijing. Journal of Insect Science, 18, https://doi.org/10.1093/jisesa/iey065.

Xun EN, Zhao JM, Guo JX, Zhang YW (2017) Nectar-dwelling microorganisms and their ecological functions. Acta Ecologica Sinica, 37, 1757-1768. (in Chinese with English abstract) [荀二娜, 赵骥民, 郭继勋, 张彦文 (2017) 花蜜 微生物及其生态功能研究进展. 生态学报, 37, 17571768.]

Yang Y, Ma S, Yan Z, Liu F, Diao Q, Dai P (2019) Effects of three common pesticides on survival, food consumption and midgut bacterial communities of adult workers Apis cerana and Apis mellifera. Environmental Pollution, 249, 860-867.

Zheng H, Nishida A, Kwong WK, Koch H, Engel P, Steele MI, Moran NA (2016) Metabolism of toxic sugars by strains of the bee gut symbiont Gilliamella apicola. mBio, 7, e01326-16.

Zou Y, Bianchi FJJA, Jauker F, Xiao H, Chen J, Cresswell J, Luo A, Huang J, van der Werf W (2017) Landscape effects on pollinator communities and pollination services in small-holder agroecosystems. Agriculture, Ecosystems and Environment, 246, 109-116.

(责任编辑：黄祥忠) 